\title{
Article
}

\section{Pseudomonas donghuensis HYS gtrA/B/II Gene Cluster Contributes to Its Pathogenicity toward Caenorhabditis elegans}

\author{
Yaqian Xiao, Panning Wang, Xuesi Zhu and Zhixiong Xie * \\ Hubei Key Laboratory of Cell Homeostasis, College of Life Sciences, Wuhan University, Wuhan 430072, China; \\ 2018102040012@whu.edu.cn (Y.X.); 2015202040019@whu.edu.cn (P.W.); 2019202040032@whu.edu.cn (X.Z.) \\ * Correspondence: zxxie@whu.edu.cn
}

check for updates

Citation: Xiao, Y.; Wang, P.; Zhu, X.; $\mathrm{Xie}, \mathrm{Z}$. Pseudomonas donghuensis HYS gtrA/B / II Gene Cluster Contributes to Its Pathogenicity toward Caenorhabditis elegans. Int. J. Mol. Sci. 2021, 22, 10741. https://doi.org/ 10.3390/ijms221910741

Academic Editor: Jorge H. Leitão

Received: 22 August 2021

Accepted: 30 September 2021

Published: 4 October 2021

Publisher's Note: MDPI stays neutral with regard to jurisdictional claims in published maps and institutional affiliations.

Copyright: (c) 2021 by the authors. Licensee MDPI, Basel, Switzerland. This article is an open access article distributed under the terms and conditions of the Creative Commons Attribution (CC BY) license (https:// creativecommons.org/licenses/by/ $4.0 /)$.

\begin{abstract}
Pseudomonas donghuensis HYS is more virulent than P. aeruginosa toward Caenorhabditis elegans but the mechanism underlying virulence is unclear. This study is the first to report that the specific gene cluster $g \operatorname{tr} A / B / I I$ in P. donghuensis HYS is involved in the virulence of this strain toward C. elegans, and there are no reports of GtrA, GtrB and GtrII in any Pseudomonas species. The pathogenicity of $P$. donghuensis HYS was evaluated using C. elegans as a host. Based on the prediction of virulence factors and comparative genomic analysis of $P$. donghuensis HYS, we identified 42 specific virulence genes in P. donghuensis HYS. Slow-killing assays of these genes showed that the $\operatorname{gtr} A B$ mutation had the greatest effect on the virulence of $P$. donghuensis HYS, and GtrA, GtrB and GtrII all positively affected $P$. donghuensis HYS virulence. Two critical GtrII residues ( $\mathrm{Glu}^{47}$ and $\mathrm{Lys}^{480}$ ) were identified in P. donghuensis HYS. Transmission electron microscopy (TEM) showed that GtrA, GtrB and GtrII were involved in the glucosylation of lipopolysaccharide (LPS) O-antigen in P. donghuensis HYS. Furthermore, colony-forming unit (CFU) assays showed that GtrA, GtrB and GtrII significantly enhanced P. donghuensis HYS colonization in the gut of C. elegans, and glucosylation of LPS O-antigen and colonization in the host intestine contributed to the pathogenicity of P. donghuensis HYS. In addition, experiments using the worm mutants ZD101, KU4 and KU25 revealed a correlation between P. donghuensis HYS virulence and the TIR-1/SEK-1/PMK-1 pathways of the innate immune p38 MAPK pathway in $C$. elegans. In conclusion, these results reveal that the specific virulence gene cluster $g \operatorname{tr} A / B / I I$ contributes to the unique pathogenicity of HYS compared with other pathogenic Pseudomonas, and that this process also involves C. elegans innate immunity. These findings significantly increase the available information about GtrA/GtrB/GtrII-based virulence mechanisms in the genus Pseudomonas.
\end{abstract}

Keywords: Pseudomonas donghuensis HYS; GtrA; GtrB; GtrII; virulence; Caenorhabditis elegans

\section{Introduction}

Virulence is a pathogen's ability to cause harm or disease, involving nutrient competition and requiring self-protection, and can thereby protect them from predators and hostile environments [1,2]. When invading a host, pathogens need to respond rapidly to adverse situations, including by activating associated virulence-related programs and escaping the host immune defenses.

Pseudomonas, a genus of obligate aerobic Gram-negative bacilli, belongs to the class of Gammaproteobacteria [3-6]. This genus contains many pathogen species, including P. aeruginosa, P. putida, P. fluorescens and P. syringae [7-9]. P. donghuensis exhibits antifungal and plant-promoting activities $[10,11]$. P. donghuensis HYS exhibits a high iron-chelating capacity and secretes pyoverdine and a large quantity of 7-hydroxytropolone, a nonfluorescent substance that exerts antibacterial and antifungal effects [12-14]. P. donghuensis HYS is the type strain of this new species and can thus be used as a representative of the whole species when conducting pathogenic research. In a previous study, this strain was shown more virulent to Caenorhabditis elegans than human opportunistic pathogens $P$. aeruginosa 
PAO1 or PA14 under the same culture conditions, suggesting the possible existence of a new pathogenic mechanism [15]. However, the pathogenic mechanism of P. donghuensis HYS remains unclear.

C. elegans is used as a model organism for the analysis of pathogenic bacteria [16], as its small body size, simple body structure, short lifespan and completely sequenced genome [17-20], and this worm can interact with many known human pathogens and has a functional innate immune response [21]. C. elegans provides us with a model for studying virulence factors, pathogenetic mechanisms and host-pathogen interactions [22,23]. In our previous work, $P$. donghuensis HYS caused a high level of mortality in C. elegans in a slow-killing experiment $[13,15,16]$.

Lipopolysaccharide (LPS) is an important pathogenic factor composed of three regions: the lipid A membrane anchor, an inner core and repeating O-antigen subunits [24]. In Shigella flexneri, O-antigen glucosylation is mediated by temperate bacteriophages, which encode a three-gene cluster that contains $g \operatorname{tr} A, g \operatorname{tr} B$ and $g \operatorname{tr}[$ [type]. GtrA family members are often involved in the synthesis of cell-surface polysaccharides [25], GtrB is polyisoprenyl phosphate glycosyltransferase, and $\operatorname{Gtr}_{[\operatorname{type}}$ encodes a species-specific glucosyltransferase [26], which specifically attaches a glucosyl residue to the appropriate rhamnose of the O-antigen chain [27-29]. However, gtrA, gtrB and gtrII have not been reported in Pseudomonas.

In this study, we first used the Virulence Factor of Pathogenic Bacteria (VFDB) database to predict virulence-related genes in P. donghuensis HYS. We then screened for specific virulence genes in P. donghuensis HYS through comparative genomic analysis of whole genome sequences of $P$. donghuensis HYS and six common pathogenic Pseudomonas model strains. We thereby obtained 42 specific virulence genes in P. donghuensis HYS and found that the $g \operatorname{tr} A / B / I I$ gene cluster does not exist in all pathogenic Pseudomonas. Our work further revealed that the $g \operatorname{tr} A / B / g \operatorname{trII}$ gene cluster positively affected virulence. In addition, we tested the correlation between $P$. donghuensis HYS virulence and $C$. elegans innate immunity. Our data suggest that the $g \operatorname{tr} A$, gtrB and gtrII gene cluster plays a role in the virulence of $P$. donghuensis HYS, and this result may facilitate increased comprehension of the pathogenesis process and mechanisms of P. donghuensis, as well as of the genus Pseudomonas more generally.

\section{Results}

2.1. Screening of Specific Virulence Genes Based on Virulence Factor Prediction and Comparative Genomic Analysis

In a previous study, we found that $C$. elegans fed with $P$. donghuensis HYS had a significantly shorter lifespan than those fed with P. aeruginosa [15], which indicates that the strain is highly virulence and potentially employs a different virulence mechanism. We first predicted virulence factors of $P$. donghuensis HYS using the VFDB database. The prediction criteria were a protein sequence identity of more than $40 \%$ and a difference in length between protein sequences of less than $30 \%$. As a result, we obtained 440 genes that may be related to virulence in $P$. donghuensis HYS. Then, we performed comparative genomic analysis of whole genome sequences of the P. donghuensis HYS strain and six model strains of Pseudomonas, which were P. aeruginosa PAO1, P. aeruginosa PA14, P. putida KT2440, P. syringae B728a, P. syringae pv tomato DC3000 and P. fluorescens Pfo-1. The clustering criteria were a protein sequence identity of more than $50 \%$ and a difference in length between protein sequences of less than $30 \%$. Cluster analysis of genes and gene families revealed 1022 specific genes and 10 gene families of P. donghuensis HYS (Figure 1A,B). Among the 1022 genes, 584 specific genes were annotated by the Clusters of Orthologous Groups (COG) database. The functional classifications of specific annotated genes were mainly cellular (135 unigenes), information (105 unigenes), metabolism (251 unigenes) and poorly characterized (93 unigenes) (Figure 1C). The 440 virulence genes predicted by the VFDB database were compared with the 584 specific genes obtained by comparative genomic analysis, we screened 42 of 440 virulence genes that are also specific genes of 
P. donghuensis HYS. Therefore, these 42 genes are specific virulence genes in P. donghuensis HYS (Table S1).

A

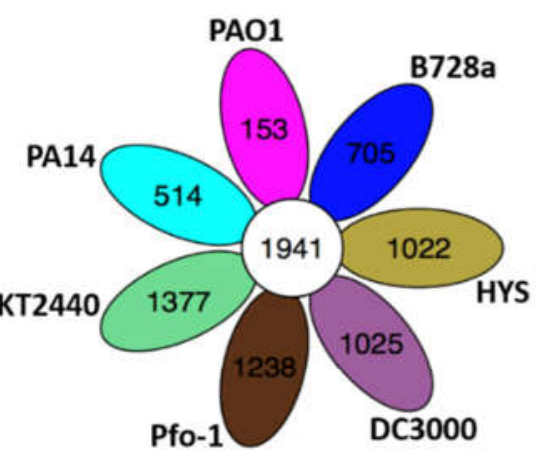

B

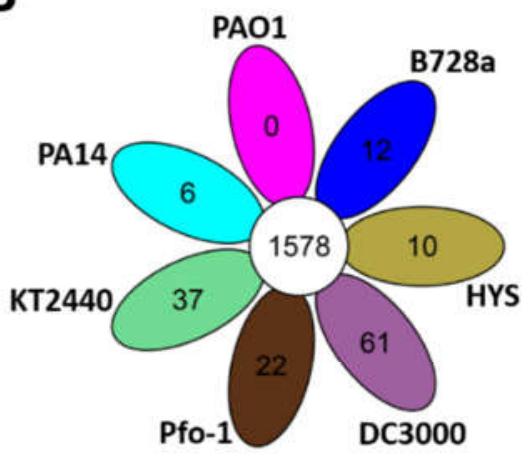

C

Cell cycle control, cell division, chromosome partitioning

Cell motility Cell wall/membrane/envelope biogenesis Defense mechanisms Extracellular structures Intracellular trafficking, secretion, and vesicular transport Posttranslational modification, protein turnover, chaperones Signal transduction mechanisms Replication, recombination and repair Transcription

Translation, ribosomal structure and biogenesis Amino acid transport and metabolism Carbohydrate transport and metabolism Coenzyme transport and metabolism Energy production and conversion Inorganic ion transport and metabolism Lipid transport and metabolism

Mobilome: prophages, transposons Nucleotide transport and metabolism Secondary metabolites biosynthesis, transport and catabolism General function prediction only

COG Function Classification

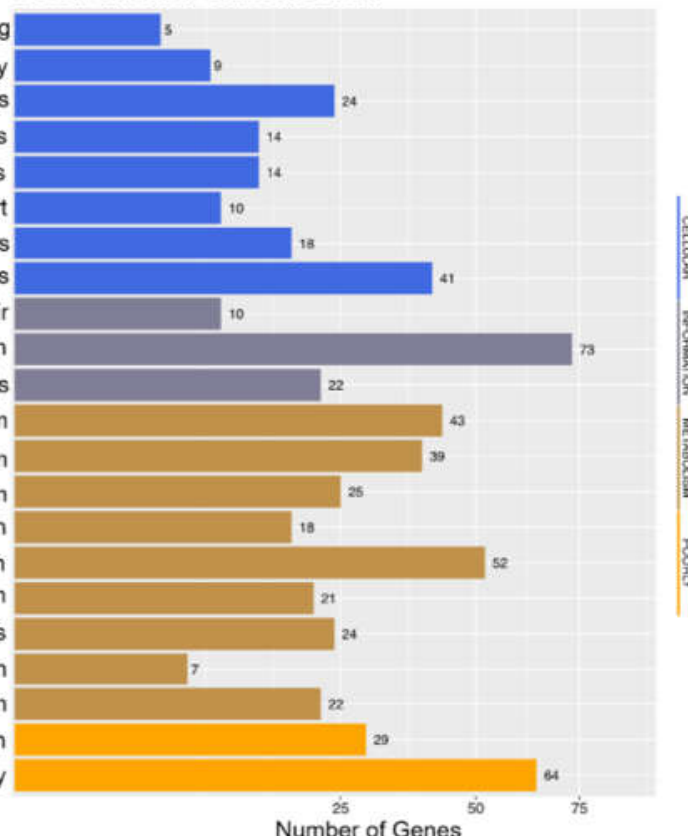

D
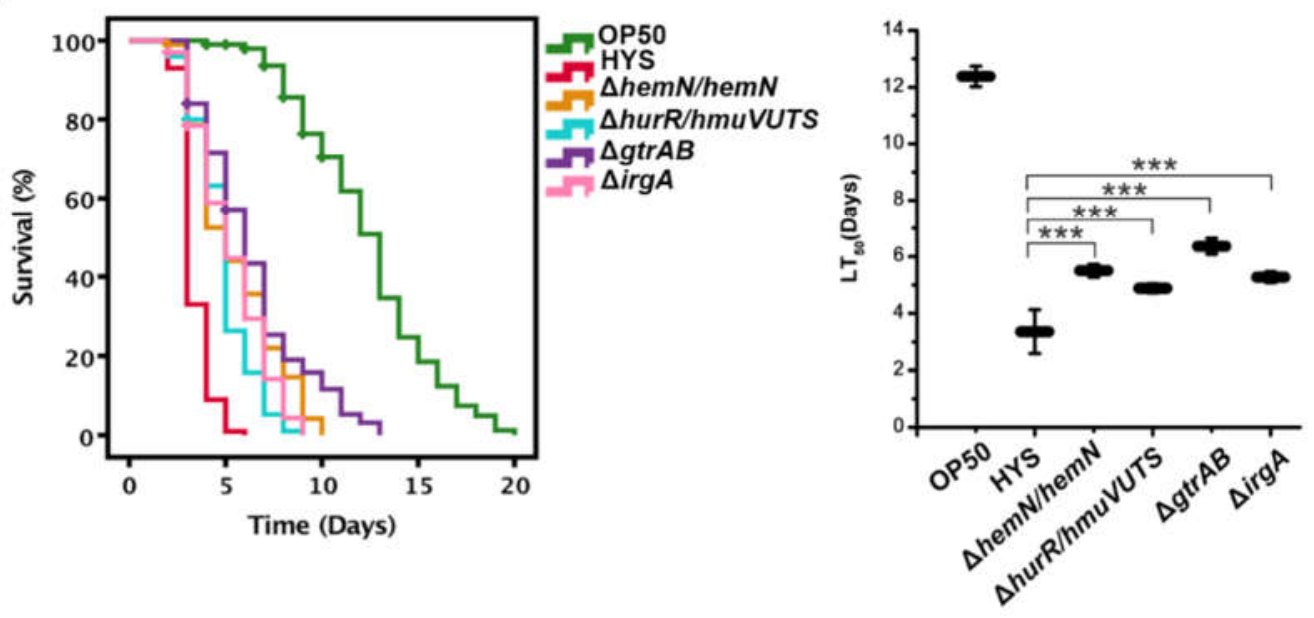

Figure 1. UpSet plots of pan gene set and the number of homologous gene families in each species. (A) UpSet plot of pan gene set among seven strains. (B) UpSet plot of the number of homologous gene families among seven strains. Each ellipse 
represents a sample, and the data in each region represent the number of clusters that only occur in samples in this region. A cluster represents a group of genes with sequence length differences of less than $30 \%$ and more than $50 \%$ similarity. (C) COG functional classification of unique genes in P. donghuensis HYS. (D) The functions of 42 unique virulence genes in P. donghuensis HYS were assessed using slow-killing experiments. Knockout strains with significant virulence reduction were listed, while other knockout strains without significant virulence reduction are listed in Figure S1. Approximately 100 synchronized adult worms were added to five plates for each bacterium. Survival curves were plotted in IBM SPSS version 18.0 using the Kaplan-Meier method. Data are presented as the mean \pm standard deviation from three independent experiments. ${ }^{* * *} p<0.001$, Student's $t$-test.

The virulence of P. donghuensis HYS is stronger than that of other Pseudomonas species, which might be related to it having these specific virulence genes. We classified and knocked out 42 specific virulence genes and performed slow-killing assays. E. coli OP50, a traditional food source for C. elegans, was used as a negative control, and wild-type HYS was used as a positive control. The survival data show that the average $\mathrm{LT}_{50}$ value (the time required to kill $50 \%$ of worms exposed to E. coli OP50) was $12.38 \pm 0.36$ days $(n=3)$. However, worms exposed to $P$. donghuensis HYS died quickly (the $\mathrm{LT}_{50}$ value decreased to $3.36 \pm 0.46$ days; $n=3$ ). By comparison, the knockout strains $\Delta$ hem $/ N, \Delta h u r R / h m u V U T S$, $\triangle g \operatorname{tr} A B$ and $\triangle \operatorname{irg} A$ had significantly reduced virulence (the $\mathrm{LT}_{50}$ values increased to 5.54 \pm 0.22 days, $4.91 \pm 0.16$ days, $6.33 \pm 0.27$ days and $5.24 \pm 0.19$ days, respectively; $n=3$ ) (Figure 1D), while other knockout strains showed no significant reductions in virulence (Figure S1). The double-knockout strain $\triangle g t r A B$ had the most significant reduction in virulence (the $\mathrm{LT}_{50}$ value was $6.33 \pm 0.27$ days; $n=3$ ), nematodes fed $\triangle g \operatorname{tr} A B$ survived for 3.0 days longer than those fed wild-type HYS (the $\mathrm{LT}_{50}$ value was $3.36 \pm 0.46$ days; $n=3$ ). Interestingly, there are no reports of GtrA and GtrB in Pseudomonas. In addition, gtrII is behind $g \operatorname{tr} B$ in the $P$. donghuensis HYS genome also found to be unique to $P$. donghuensis HYS through comparative genome analysis. However, the function of GtrII is not clear, and GtrII has not been annotated in VFDB, KEGG (Kyoto Encyclopedia of Genes and Genomes), COG or GO (Gene Ontology) databases through analyses genome sequencing data; therefore, GtrII was not screened through VFDB prediction and COG database annotation. S. flexneri has a three-gene cluster containing $g \operatorname{tr} A, g \operatorname{tr} B$ and $g \operatorname{tr}_{\text {[type] }} ; g t r_{\text {[type] }}$ encodes a species-specific glucosyltransferase, which specifically attaches a glucosyl residue to the appropriate rhamnose of the O-antigen chain. The $g \operatorname{tr} A, g \operatorname{tr} B$ and $g \operatorname{trII}$ genes may be involved in the virulence of HYS and may provide this strain with special virulence characteristics compared with pathogenic Pseudomonas.

\subsection{The Particularity of GtrA, GtrB and GtrII in Pseudomonas}

In order to obtain clues about the evolution of the gene combination, we compared the protein homology of GtrA, GtrB and GtrII using BLASTP and set the threshold at $40 \%$. The comparison results showed that most Pseudomonas, including those that are pathogenic, contain one or two genes of the $g \operatorname{tr} A / B / I I$ gene cluster, and the arrangement of GtrA/B/II only exists in four strains other than HYS (Figure 2A). Five strains, including $P$. donghuensis HYS, were isolated from different biological samples in different countries, and there is evidence of geographical isolation (Table 1). To better understand the evolutionary position of GtrA, GtrB and GtrII in P. donghuensis HYS, we constructed phylogenetic trees according to the amino acid sequences of GtrA, GtrB and GtrII in bacteria using the neighbor-joining method with a bootstrap value of 1000 (Figure 2B). The phylogenetic trees show that the GtrA/B/II cluster in P. donghuensis HYS is grouped most closely with that in P. vranovensis, followed by Pseudomonas sp. WS 5059. These results suggest that the $g \operatorname{tr} A / B / I I$ gene cluster has an evolutionary particularity, which is that the gene combination did not form by gradual evolution but formed in a short time in a changeable environment to meet the needs of bacterial survival and growth. 
A

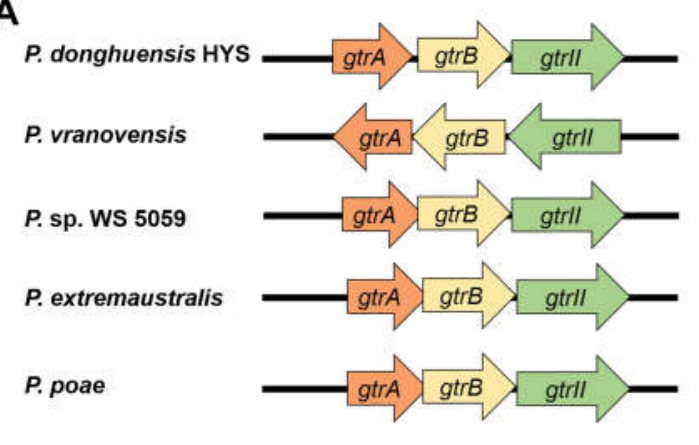

B

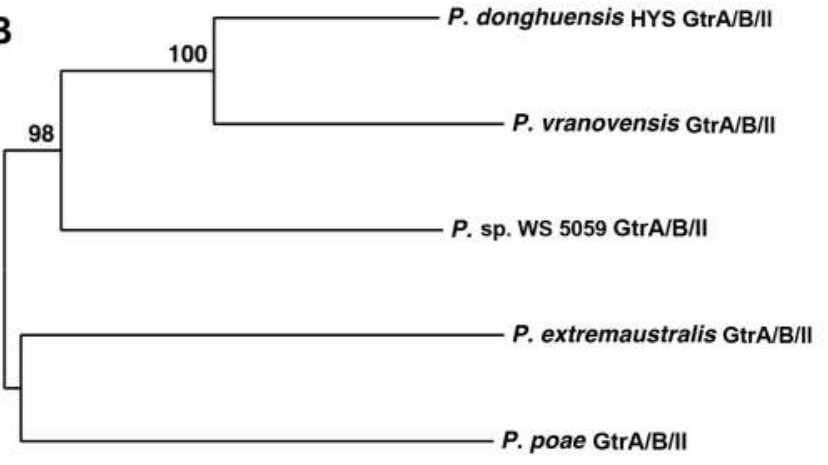

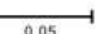

Figure 2. Arrangement and phylogenetic analysis of GtrA/B/II in Pseudomonas. (A) Arrangement of GtrA/B/II in five strains. (B) Phylogenetic analysis of P. donghuensis HYS GtrA/B/II using amino acid sequences with MEGA7.0 program.

Table 1. Information on strains with the same arrangement of GtrA/B/II as P. donghuensis HYS.

\begin{tabular}{ccccc}
\hline Species & Version & Isolation Source & Isolation Country & Reference \\
\hline $\begin{array}{c}\text { P. donghuensis HYS } \\
\text { P. vranovensis }\end{array}$ & NZ_AJJP0000000.1 & Lake & China & Gao et al., 2012 [12] \\
Pseudomonas sp. WS & NZ_JAAQWO010000002.1 & Raw milk & USA & Tao et al., 2016 [30] \\
5059 & NZ_PCQN01000008.1 & Gotting apple & Germany & Maier et al., 2020 [31] \\
P. poae & NZ_AHIP01000006.1 & $\begin{array}{c}\text { Temporary water } \\
\text { pond in Antarctica }\end{array}$ & Argentina & Tribelli et al., 2012 [33] \\
P. extremaustralis & & &
\end{tabular}

2.3. The Virulence of P. donghuensis HYS Is Optimized by the gtrA/B/II Virulence Gene Cluster in a C. elegans Slow-Killing Assay

P. donghuensis HYS is highly virulent toward C. elegans. We performed a slow-killing assay to test bacterial virulence against $C$. elegans. To test the functions of $g \operatorname{tr} A, g \operatorname{tr} B$ and gtrII in virulence, we constructed single-knockout strains $\Delta g \operatorname{tr} A, \Delta g \operatorname{tr} B$ and $\Delta g \operatorname{tr} I I$, the double-knockout strain $\triangle g \operatorname{tr} A B$ and the triple-knockout strain $\triangle g \operatorname{tr} A B I I$ and performed a slow-killing assay utilizing C. elegans. Survival analysis showed that the lifespan and $\mathrm{LT}_{50}$ value of $C$. elegans fed the single-knockout strains were approximately 1.5 times greater than those fed the wild-type strain (the $\mathrm{LT}_{50}$ values increased to $4.39 \pm 0.13$ days, $4.77 \pm 0.16$ days and $5.09 \pm 0.19$ days, respectively; $n=3$ ); those fed the double-knockout strain $\triangle g t r A B$ and the triple-knockout strain $\triangle g \operatorname{tr} A B I I$ survived for approximately twice as long as those fed the wild-type strain (the $\mathrm{LT}_{50}$ values increased to $6.33 \pm 0.27$ days and $6.02 \pm 0.23$ days, respectively; $n=3$ ) (Figure $3 \mathrm{~A}$ ). 
A
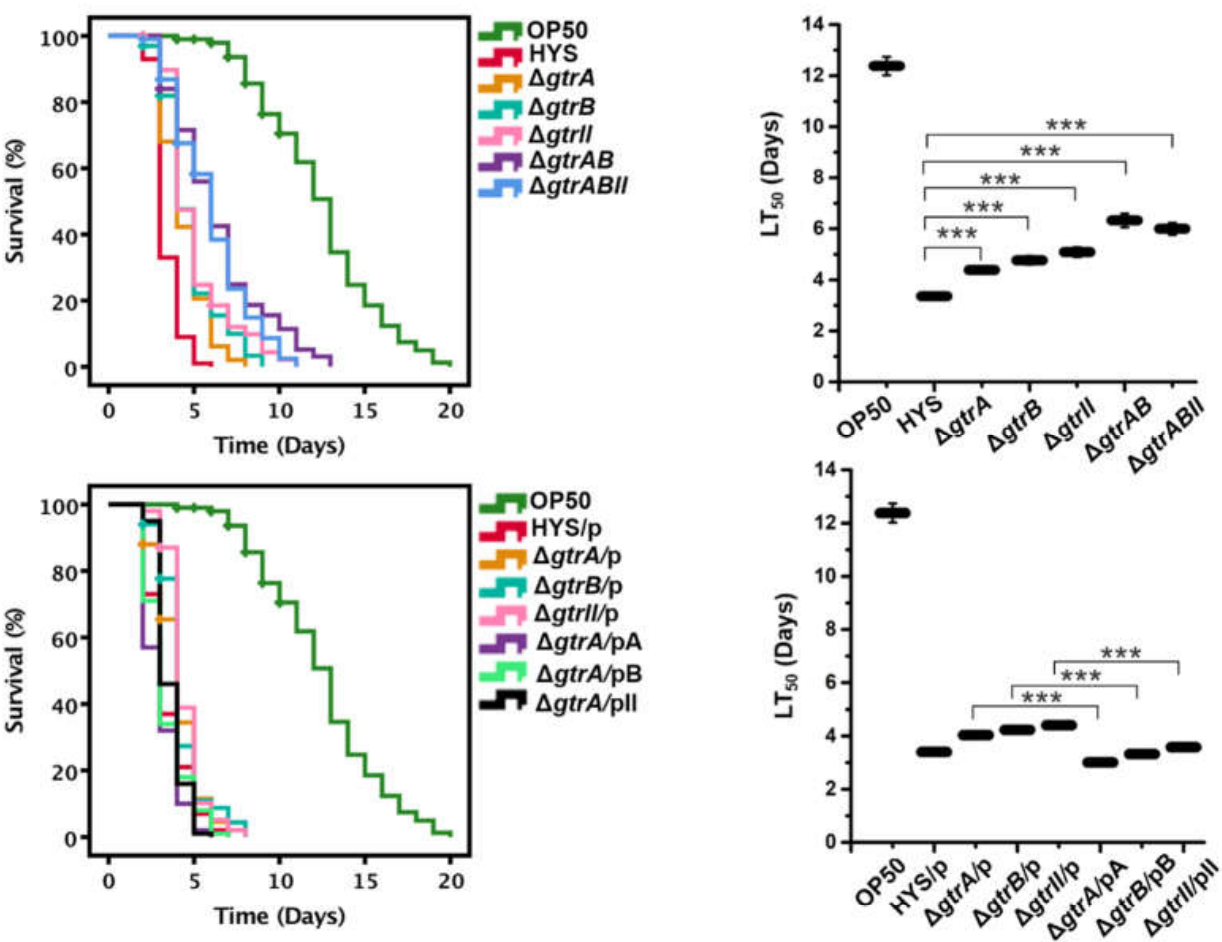

C
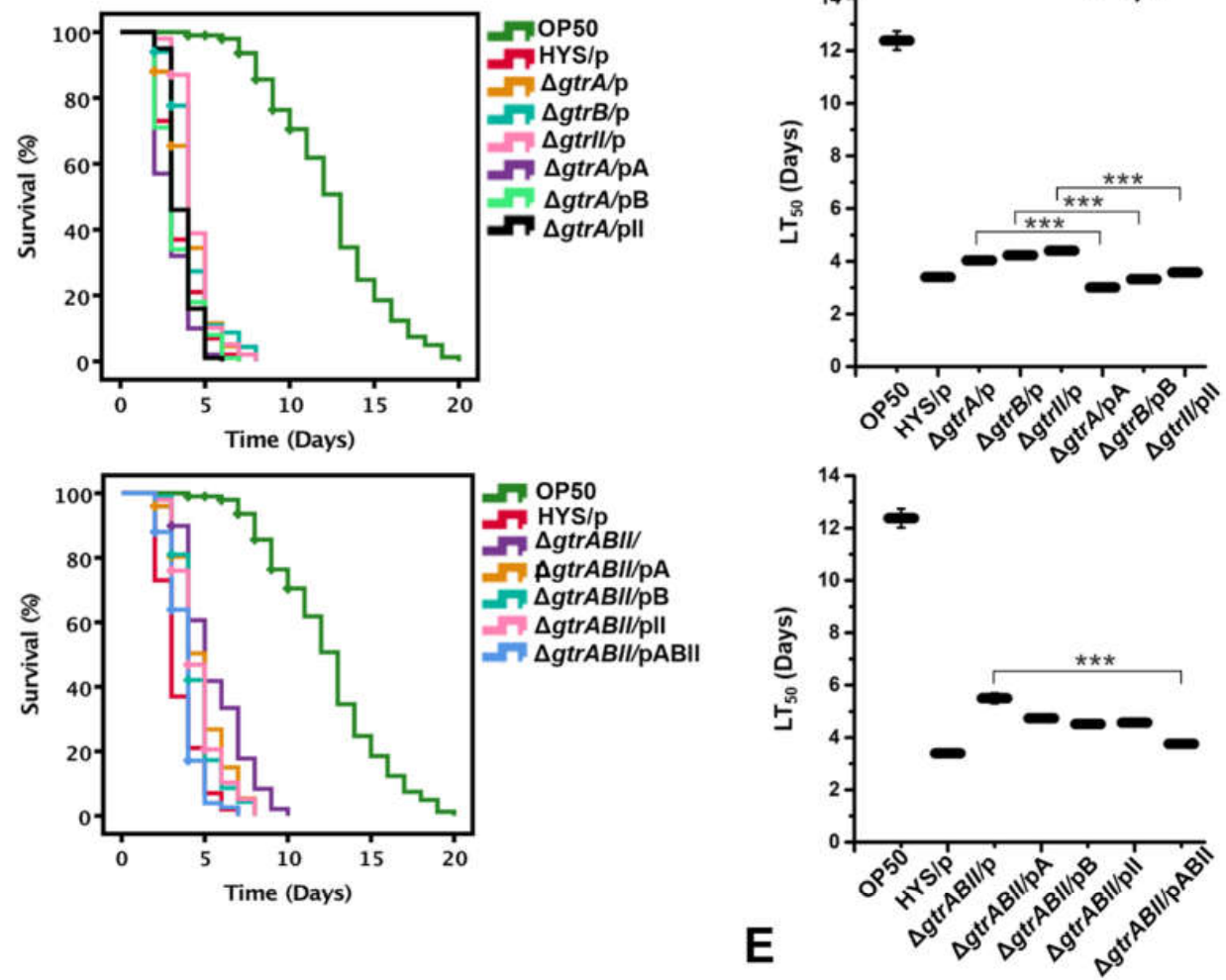

D
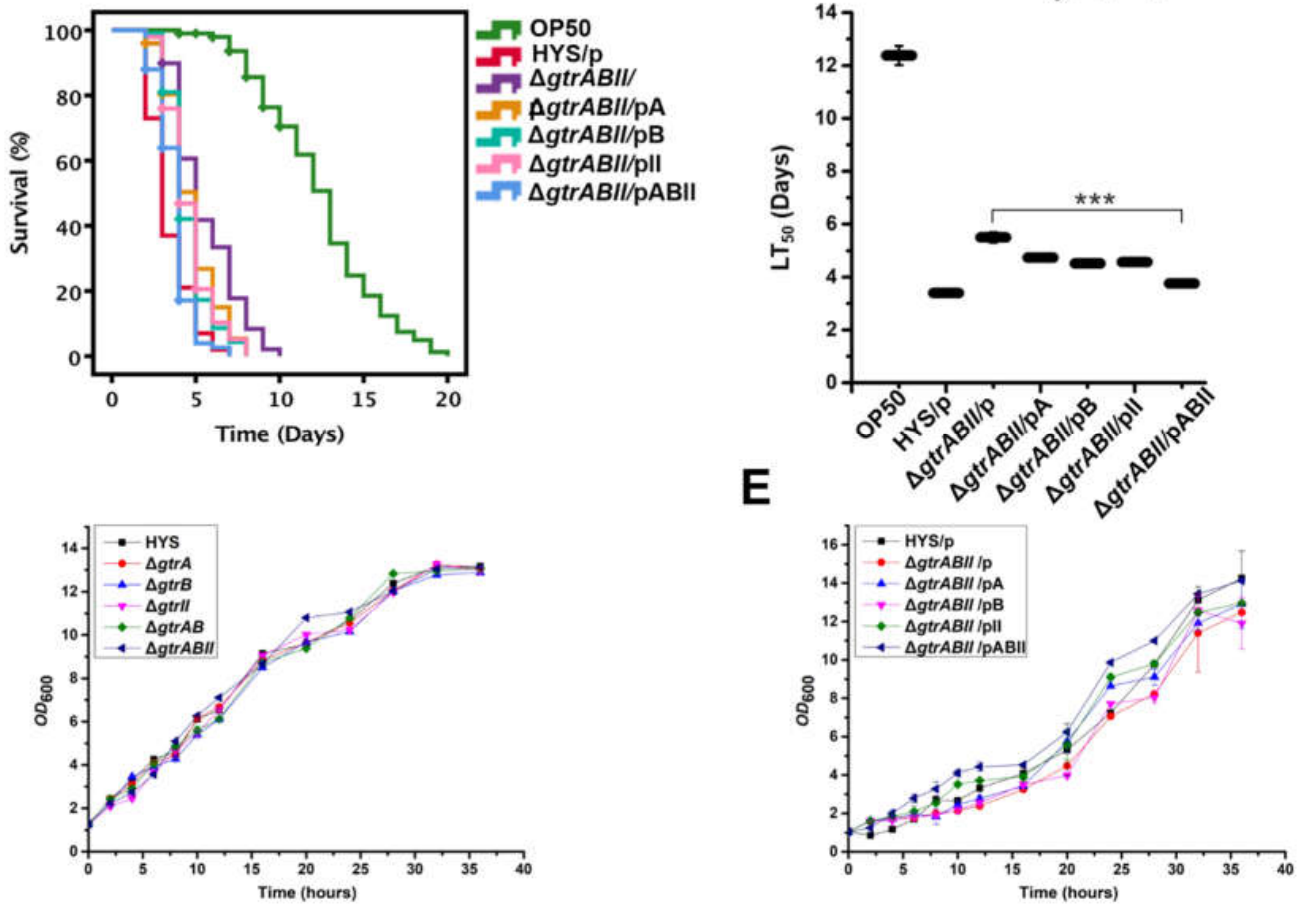

Figure 3. The $g \operatorname{tr} A / B / I I$ gene cluster is associated with pathogenicity in the C. elegans slow-killing model. (A) Mutants of $g \operatorname{tr} A, g \operatorname{tr} B$ and $g \operatorname{trII}$ were tested using slow-killing experiments. (B) The functions of $g \operatorname{tr} A, g \operatorname{tr} B$ and $g \operatorname{trII}$ were further confirmed by using single-gene complementation in the single-knockout strains. $p$ represents the expression plasmid pBBR1MCS-2; pA, pB and pII represent the recombinant plasmids pBBR2-gtrA, pBBR2-gtrB and pBBR2-gtrII, respectively. (C) We also performed single-gene complementation and three-gene complementation in the triple-knockout strains. pABII represents the recombinant plasmid pBBR2-gtrABII. Approximately 100 synchronized adult worms were added to five plates for each bacterium. Survival curves were plotted in IBM SPSS version 18.0 using the Kaplan-Meier method. (D,E) Growth curves of P. donghuensis HYS and mutants. Data are presented as the mean \pm standard deviation from three independent experiments. ${ }^{* * *} p<0.001$, Student's $t$-test. 
We performed single-gene complementation in the single-knockout strains and conducted a slow-killing assay. Restoration of virulence in the complemented strains further confirmed the functions of these three genes in bacterial virulence (the $\mathrm{LT}_{50}$ values decreased from 4.04 $\pm 0.13,4.23 \pm 0.14$ and $4.41 \pm 0.10$ days to $3.01 \pm 0.10,3.32 \pm 0.12$ and $3.58 \pm 0.08$ days, respectively; $n=3$ ) (Figure $3 \mathrm{~B}$ ). In addition, we also performed single-gene complementation and three-gene complementation in the triple-knockout strain $\triangle g t r A B I I$, and the complemented strains were assayed in the slow-killing experiment. Survival analysis showed that single-gene complementation in the triple-knockout strain $\triangle g t r A B I I$ resulted in the slight restoration of virulence but could not return it to the level of the wild-type strain. Three-gene complementation in the triple-knockout strain $\triangle g \operatorname{tr} A B I I$ could restore virulence to the wild-type level (the $\mathrm{LT}_{50}$ values decreased from $5.50 \pm 0.19$ to $3.61 \pm 0.10$ days; $n=3$ ) (Figure 3C). These results further confirm that $g \operatorname{tr} A, g \operatorname{tr} B$ and $g \operatorname{tr} I I$ form a virulence gene cluster. In addition, we tested the growth of the mutants, and it was found that the growth trend of the knockout strains was basically the same as that of the wild-type strain, without obvious growth defects (Figure 3D,E). Thus, the reduced virulence was not due to a deficiency in bacterial growth, which demonstrated that the reduced virulence of the knockout strains was indeed caused by the deletion of gene functions. These results suggest that the $g \operatorname{tr} A / B / I I$ gene cluster plays a crucial role in the virulence of $P$. donghuensis HYS.

\subsection{Identification of Critical GtrII Residues in P. donghuensis HYS}

To further understand the virulence effect of GtrII in P. donghuensis HYS, the GtrII protein sequence of $P$. donghuensis HYS was compared with BLASTP in NCBI, which showed that GtrII belonged to the GtrII protein superfamily and was a transmembrane protein with nine transmembrane regions (Figure S2). In S. flexneri, three critical residues (Glu ${ }^{40}$, Phe ${ }^{414}$, and Lys ${ }^{478}$ ) are conserved in Gtr $_{\text {[type] }}$ [34]. However, Bioinformatics was limited in finding conserved residues between them as a result of the low sequence homology and different protein lengths among $G \operatorname{tr}_{[\text {type] }}$. Based on the conservative localization of critical residues among Gtr ${ }_{\text {[type] }}$, GtrII residues $\left(\mathrm{Glu}^{47}\right.$, Phe ${ }^{430}$, Phe ${ }^{431}$ and Lys ${ }^{480}$ ) were manually identified and selected for point mutating to alanine (Figure S3). Accordingly, we constructed strains with E47A, F430A, F431A and K480A point mutations in the genome and performed a slow-killing assay to test the virulence of the mutants against $C$. elegans. The results show that the virulence function of GtrII was abolished in the E47A and K480A mutants (the $\mathrm{LT}_{50}$ values increased from $3.36 \pm 0.46$ days to $5.01 \pm 0.17$ days and $5.05 \pm 0.14$ days, respectively; $n=3$ ) (Figure $4 \mathrm{~A}$ ), which is consistent with $\Delta g \operatorname{trII}$ (the $\mathrm{LT}_{50}$ value was $5.09 \pm 0.19$ days; $n=3$ ). F430A and F431A mutants did not show the same reduced virulence as $\Delta g t r I I$. In order to exclude the possible influence of the growth of the point mutation strains on virulence, we tested the growth of the mutants. The growth trend of the point mutation strains was found to be basically the same as that of the wild-type strain, without obvious growth defects (Figure 4B). A colony-forming unit (CFU) assay was performed to determine the colonization of point mutation strains in the gut of C. elegans (Figure 4C). E47A and K480A mutations were observed to significantly inhibit the number of living bacteria colonized in the gut of $C$. elegans. However, we observed that F430A and F431A mutations did not inhibit P. donghuensis HYS colonization in C. elegans. These results show that two critical GtrII residues (Glu47 and Lys480) control the virulence function of GtrII in P. donghuensis HYS. At present, there are no reports describing the role of GtrII in bacterial virulence. 
A

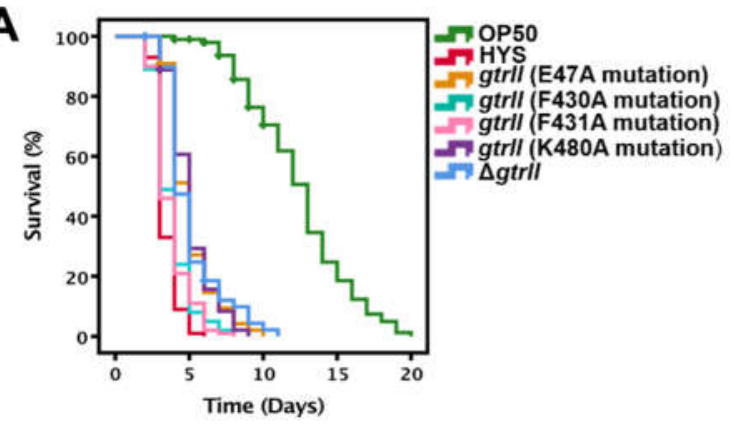

B

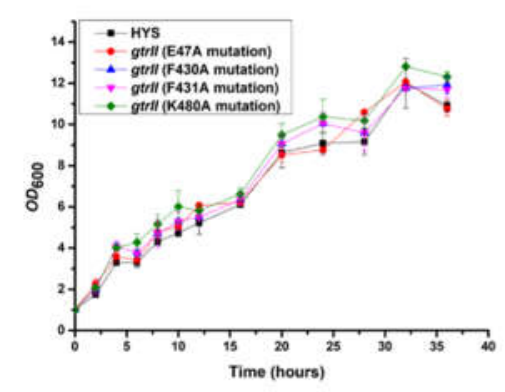

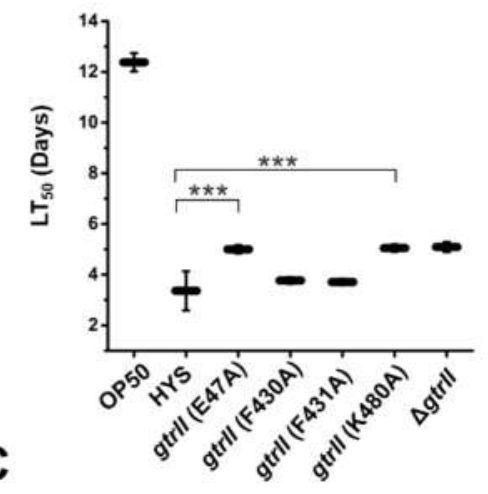

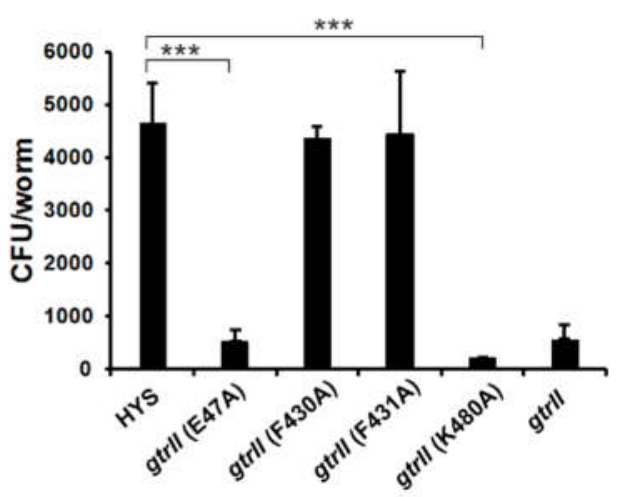

Figure 4. Critical GtrII residues control the virulence function of GtrII in P. donghuensis HYS. (A) The effects of E47A, F430A, F431A and K480A point mutations in P. donghuensis HYS were tested using slow-killing experiments. Approximately 100 synchronized adult worms were added to five plates for each bacterium. Survival curves were plotted in IBM SPSS version 18.0 using the Kaplan-Meier method. (B) Growth curves were also constructed in this killing experiment. (C) P. donghuensis HYS CFU in the gut of nematodes exposed to point mutation strains. Data are presented as the mean \pm standard deviation from three independent experiments. ${ }^{* * *} p<0.001$, Student's $t$-test.

\subsection{Surface Topology of Strains Revealed by Transmission Electron Microscopy}

To test the correlation between $g \operatorname{tr} A$, gtrB and gtrII and the glucosylation of LPS Oantigen, bacteria were visualized by transmission electron microscopy (TEM) (Figure 5). Wild-type HYS had dense surface material extending about $20 \mathrm{~nm}$ beyond the outer membrane (between the two arrowheads). By contrast, the exteriors of $\triangle g \operatorname{tr} A, \Delta g \operatorname{tr} B, \Delta g \operatorname{trII}$, $\triangle g \operatorname{tr} A B$ and $\triangle g \operatorname{tr} A B I I$ were more diffuse. The filamentous material of $\triangle g \operatorname{tr} A$ extended around $34 \mathrm{~nm}$ from the outer membrane, and the filamentous material of $\triangle g \operatorname{tr} B, \Delta g \operatorname{trII}$, $\triangle g \operatorname{tr} A B$ and $\triangle g \operatorname{tr} A B I I$ extended around $40 \mathrm{~nm}$ from the outer membrane. $\triangle g \operatorname{tr} A B I I / \mathrm{pABII}$ had dense surface material extending around $20 \mathrm{~nm}$ from the outer membrane, which was basically the same as that of the wild-type strain. The glucosylation of LPS O-antigen in P. donghuensis HYS dramatically shortens the $\mathrm{O}$ antigen, halving the distance that it extends beyond the outer membrane. GtrA, GtrB and GtrII may be involved in the glucosylation of LPS O-antigen in P. donghuensis HYS. 


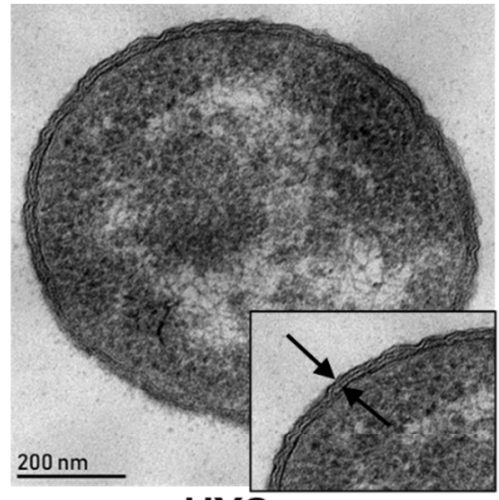

HYS

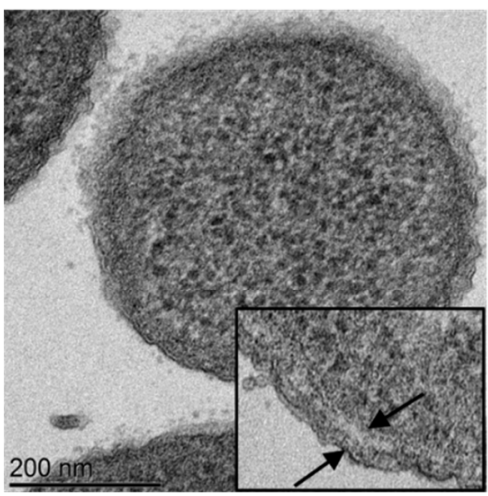

$\Delta g t r l l$

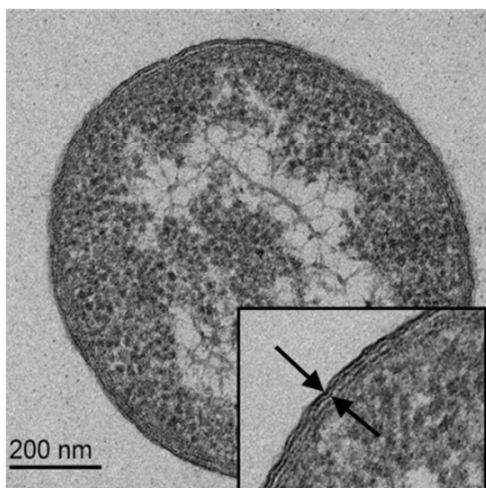

$\Delta g$ trABII /pABII

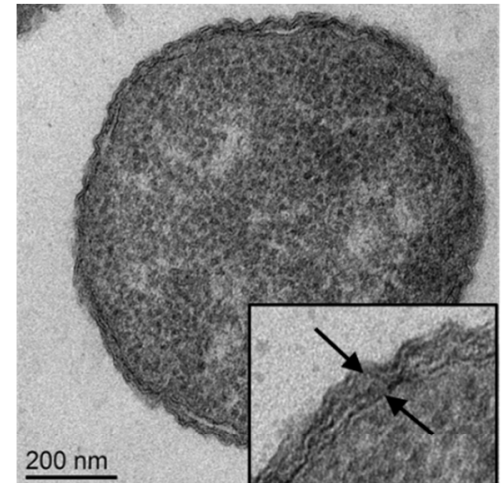

$\Delta g t r A$

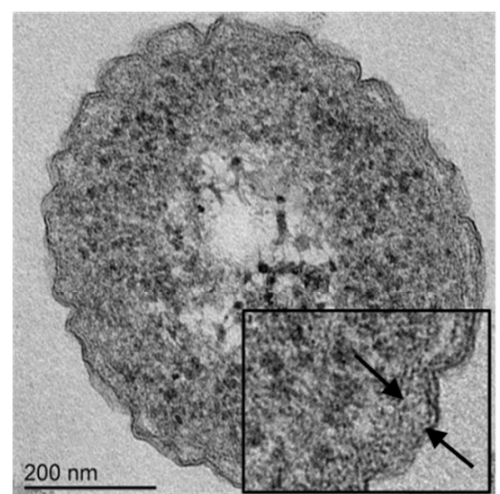

$\Delta g \operatorname{tr} A B$

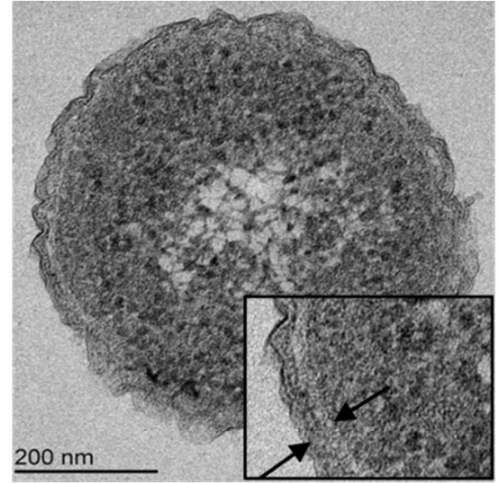

$\Delta g t r B$

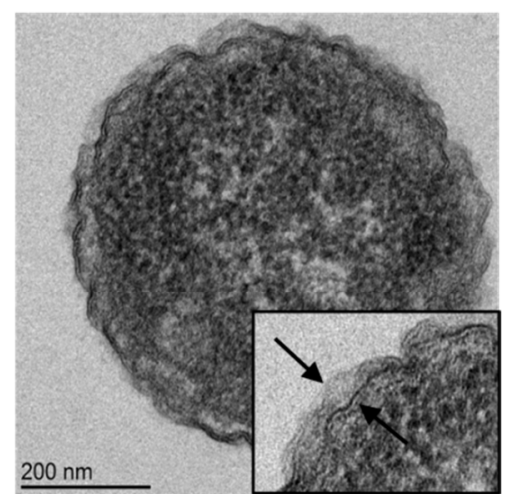

$\Delta g$ trABII

Figure 5. Surface topology of strains revealed by transmission electron microscopy after treatment with ruthenium red. The electron-dense material on the bacterial surface is indicated between the arrows. In $\triangle g \operatorname{tr} A, \Delta g \operatorname{tr} B, \Delta g \operatorname{trII}, \Delta g \operatorname{tr} A B$ and $\triangle g \operatorname{tr} A B I I$, the surface material is less compact than HYS.

\subsection{Estimation of P. donghuensis HYS CFU within the C. elegans Gut}

C. elegans was exposed to P. donghuensis HYS in the same conditions as those of the slow-killing assay. We measured CFU to assess the colonization of deletion mutants in the gut of C. elegans (Figure 6). To rule out the possibility of an effect of different pharyngeal pumping rates on bacterial load, we quantitatively measured pathogen-clearance ability by first ensuring that $\mathrm{N} 2$ worms were inoculated with a similar number of bacteria. We observed that deletion mutants of $g \operatorname{tr} B, g \operatorname{tr} I I, g \operatorname{tr} A B$ and $g \operatorname{tr} A B I I$ significantly suppressed $P$. donghuensis HYS colonization in the gut of nematodes. The deletion mutant of $g t r A$ also significantly inhibited the intestinal colonization of $P$. donghuensis HYS, but the effect was weaker than that of the other mutants. Colonization in the host is the first and key step of 
exerting pathogenicity for a bacterium. These results suggest that the involvement of GtrA, GtrB and GtrII in bacterial virulence may be related to the colonization of bacteria in the host gut.

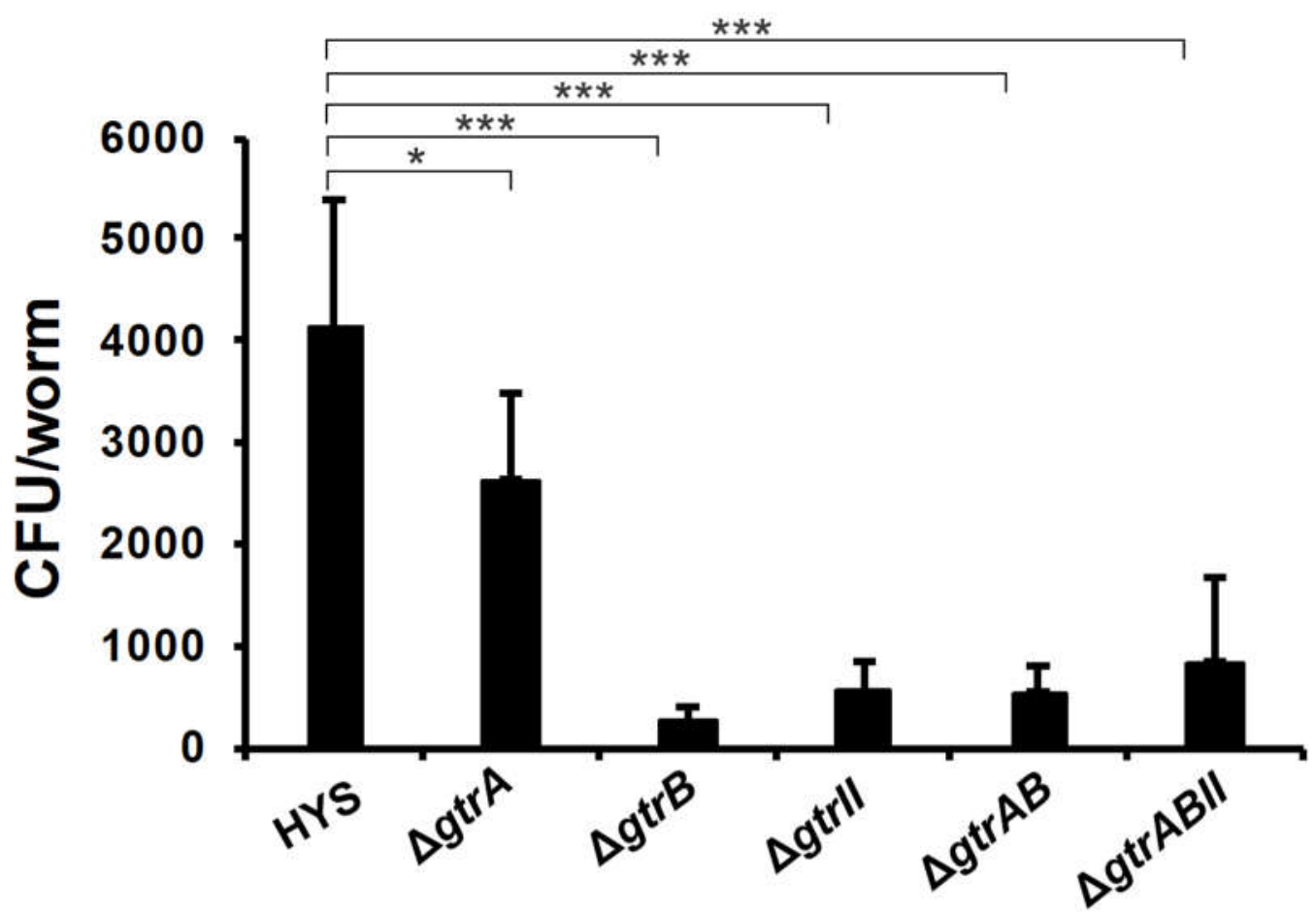

Figure 6. P. donghuensis HYS CFU in the gut of nematodes exposed to deletion mutants. Each symbol represents the average of 6 biological replicates of 10 worms. A comparable initial inoculum was ensured for the exposure of wild-type worms to HYS, $\Delta g \operatorname{tr} A, \Delta g \operatorname{tr} B, \Delta g \operatorname{trII}, \Delta g \operatorname{tr} A B$ and $\triangle g t r A B I I$ for $24 \mathrm{~h}$. Data are presented as the mean \pm standard deviation from three independent experiments. ${ }^{*} p<0.05$ and ${ }^{* * *} p<0.001$, Student's $t$-test.

\subsection{GtrA/B/II-Involved Virulence Is Related to C. elegans Innate Immunity}

Unlike higher organisms, C. elegans lacks adaptive immune pathways, and only innate immune pathways play an important role in resisting pathogens, oxidative stress and other types of stress [21]. To investigate the worm response to P. donghuensis HYS virulence, we utilized relevant mutants to conduct a slow-killing assay. The mutant worms ZD101, KU4 and KU25 are deficient in the Tir-1, SEK-1 and PMK-1 pathways of the p38 MAPK pathway, respectively. After feeding on P. donghuensis HYS, mutant C. elegans worms were more sensitive and died more quickly than N2 worms. However, when we used the gtr $A B$ deletion mutant as an alternative food source, their viability was significantly improved, illustrating that the strain $\triangle g \operatorname{tr} A B$ had lower virulence than the wild-type strain when tested as a food source for mutant worms. The $\mathrm{LT}_{50}$ values of mutant worms ZD101 (Figure 7A), KU4 (Figure 7B) and KU25 (Figure 7C) fed P. donghuensis HYS were approximately 49.25\%, $64.78 \%$ and $33.13 \%$ lower than that of $\mathrm{N} 2$ worms fed P. donghuensis HYS (the $\mathrm{LT}_{50}$ value decreased from $3.35 \pm 0.07$ to $1.69 \pm 0.04,1.18 \pm 0.03$ and $2.24 \pm 0.05$ days, respectively; $n=3)$. In addition, when the $\triangle g \operatorname{tr} A B$ deletion mutant was provided as an alternative food source, the $\mathrm{LT}_{50}$ values of mutant worms decreased by approximately $54.66 \%, 58.17 \%$ and $49.52 \%$ compared with that of $\mathrm{N} 2$ worms (the $\mathrm{LT}_{50}$ value decreased from $6.24 \pm 0.22$ days to $2.82 \pm 0.05$ days, $2.61 \pm 0.08$ days and $3.15 \pm 0.10$ days, respectively; $n=3$ ). These percentages suggest a correlation between TIR-1/SEK-1/PMK-1 of the innate immunity p38 MAPK pathway in C. elegans and the gtrAB-related virulence of $P$. donghuensis HYS. 
A
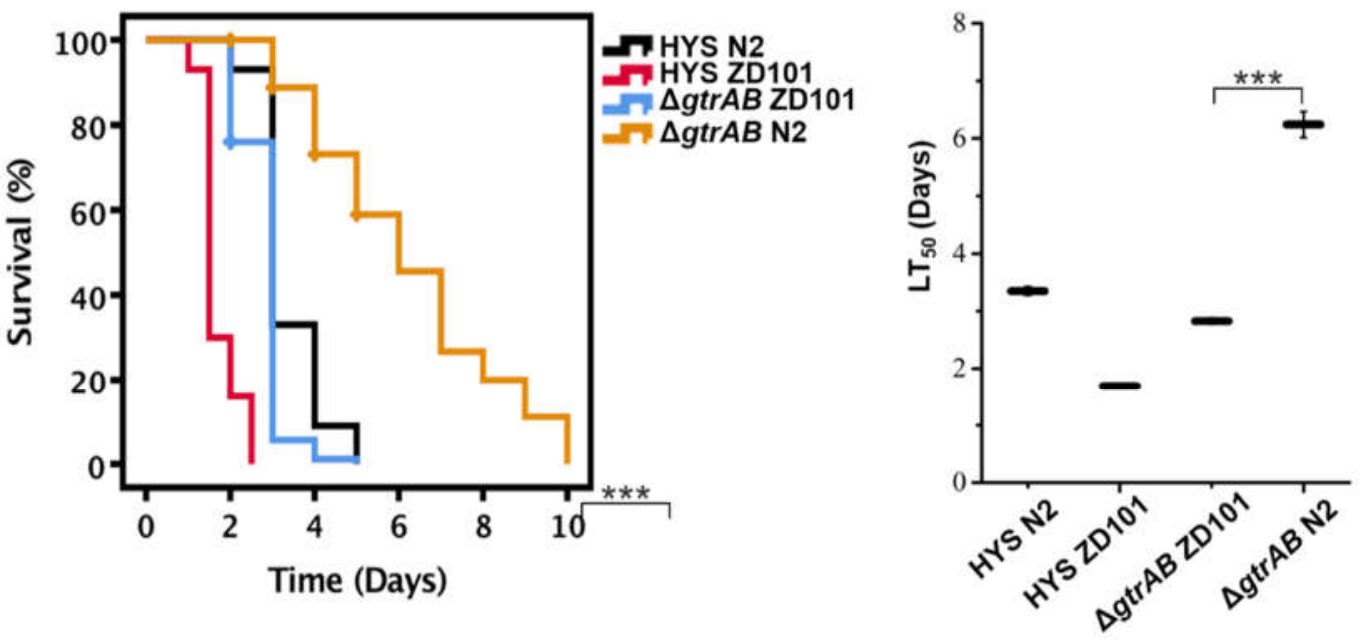

B
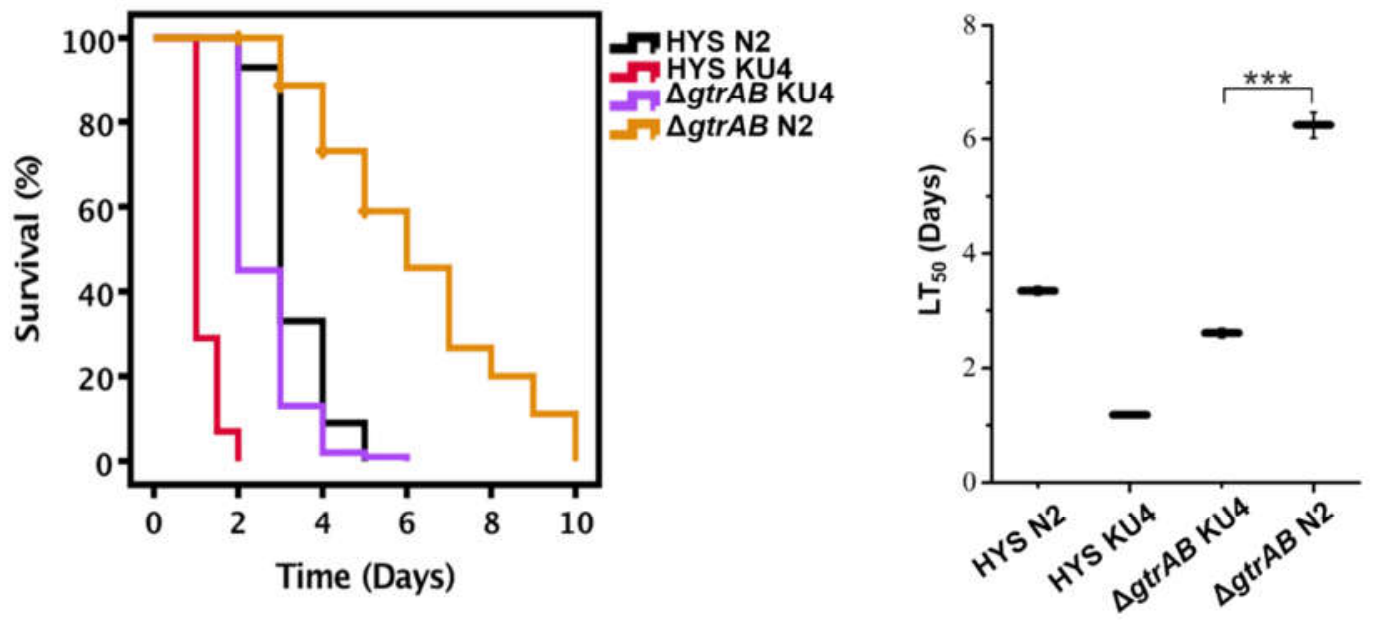

C
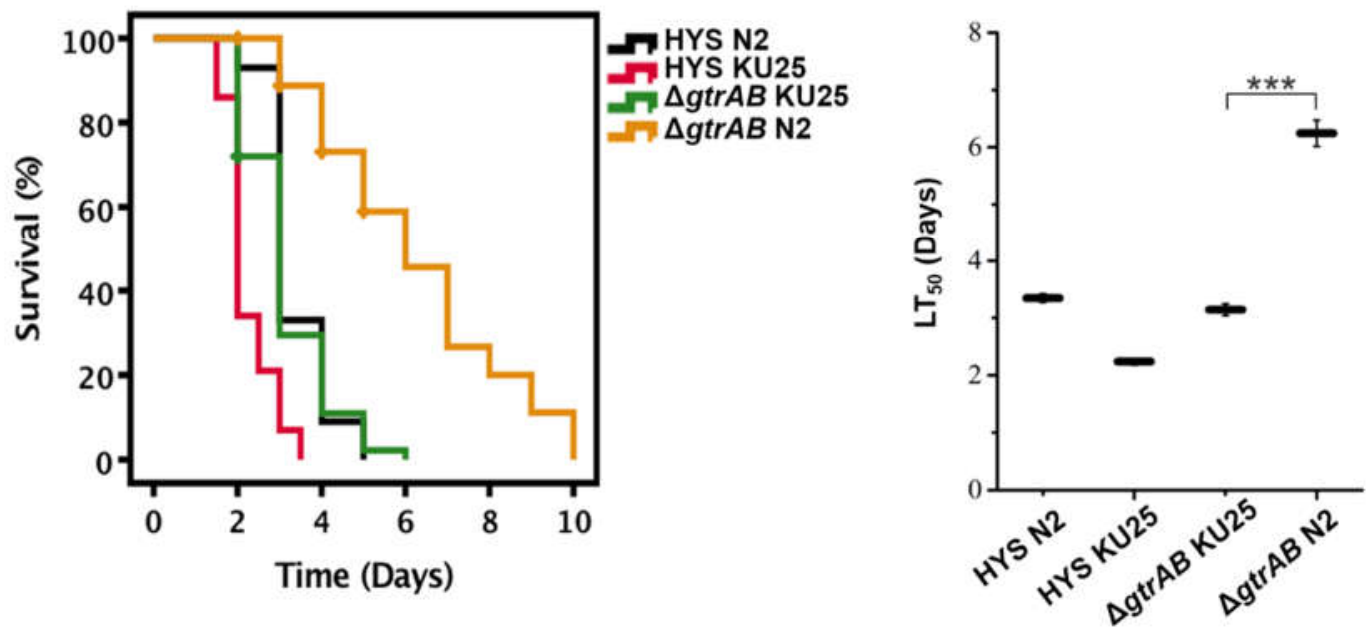

Figure 7. Correlation between GtrA/B/II-involved virulence and worm innate immunity. (A) N2 and mutant worms deficient in the Tir-1 pathway, (B) the SEK-1 pathway and (C) the PMK-1 pathway of the p38 MAPK pathway were fed P. donghuensis HYS or gtrAB. Approximately 100 synchronized adult worms were added to five plates for each bacterium. Survival curves were plotted in IBM SPSS version 18.0 using the Kaplan-Meier method. Data are presented as the mean \pm standard deviation from three independent experiments. ${ }^{* * *} p<0.001$, Student's $t$-test. 


\section{Discussion}

The virulence of $P$. donghuensis HYS is stronger than that of pathogenic P. aeruginosa PAO1 or P. aeruginosa PA14 [15]. Based on bioinformatics analysis and functional identification, we identified gtrA/B/II as a specific virulence gene cluster in P. donghuensis HYS compared with other pathogenic Pseudomonas, being found to exists in only four strains other than $P$. donghuensis HYS. A slow-killing assay confirmed that $g \operatorname{tr} A$, gtrB and $g \operatorname{trII}$ form a virulence gene cluster, and only in the presence of all three genes will it recover its virulence effects. GtrA family members are often involved in the synthesis of cell-surface polysaccharides [25], and GtrB is polyisoprenyl phosphate glycosyltransferase.Gtr ${ }_{\text {[type] }}$ encodes a species-specific glucosyltransferase [26], which specifically attaches a glucosyl residue to the appropriate rhamnose of the $\mathrm{O}$-antigen chain $[27,28]$. At present, the virulence function of GtrII is unclear, and it has not been annotated in many databases. The GtrII protein belongs to the GtrII protein superfamily and is a transmembrane protein with nine transmembrane regions. Currently, $\mathrm{Gtr}_{\text {[type] }}$ has only been reported in S. flexneri and is involved in the modification of the O-antigen. However, bioinformatics was of limited use in finding conserved residues between them as a result of the low sequence homology and different protein lengths among $\operatorname{Gtr}_{[\mathrm{type}]}$. To further understand the function of GtrII, the residues $\mathrm{Glu}^{47}$, Phe ${ }^{430}$, Phe ${ }^{431}$ and Lys ${ }^{480}$ were manually selected for E47A, F430A, F431A and K480A mutagenesis, respectively. The E47A and K480A mutations were found to abolish the virulence function of GtrII in P. donghuensis HYS, while the F430A and F431A mutations did not completely eliminate the virulence function of GtrII. We found that changing critical residues $\left(\mathrm{Glu}^{47}\right.$ and Lys ${ }^{480}$ ) did not affect the structure of the GtrII protein. However, changing critical residues affected the colonization of the mutants and thus affected their virulence.

In S. flexneri 5a, gtrA, gtrB and $g \operatorname{tr} V$ are involved in the glucosylation of LPS Oantigen $[29,34,35]$. Glucosylation of LPS O-antigen induces a transition from a linear to helical comformation with the glucosyl residue exposed to the outside of the helix, forming a more compact structure than unglucosylated LPS [36]. This dramatically shortens the $\mathrm{O}$ antigen, halving the distance that it extends beyond the outer membrane. In the LPS model, the $\mathrm{O}$ antigen is only $11 \mathrm{~nm}$ under the action of glucosylation, while in the absence of glucosylation, the $\mathrm{O}$ antigen would extend about $21 \mathrm{~nm}$ beyond the outer membrane. In S. flexneri, the exterior of M90T is a dense material extending around $35 \mathrm{~nm}$ from the outer membrane. In contrast, M90T $\Delta g t r$ has more diffuse material extending about $70 \mathrm{~nm}$ beyond the outer membrane [35]. In P. donghuensis HYS, wild-type HYS has dense surface material extending about $20 \mathrm{~nm}$ beyond the outer membrane. By contrast, the exteriors of mutants are more diffuse material extending around $40 \mathrm{~nm}$ from the outer membrane. In addition, the surface of $\triangle g t r A B I I / p A B I I$ was basically the same as that of the wildtype HYS, filamentous material that extended around $20 \mathrm{~nm}$ beyond the outer membrane. Glucosylation of LPS O antigen dramatically shortening of the $\mathrm{O}$ antigen, also halving the distance that it extends beyond the outer membrane. These results are consistent with the results of glycosylation of S. flexneri O-antigen [36,37], indicating that GtrA, GtrB and GtrII may be involved in the glycosylation of LPS O-antigen. These results are consistent with the toxicity test results, indicating that the involvement of GtrA, GtrB and GtrII in bacterial toxicity may be related to the glycosylation of LPS O-antigen.

Colonization in the host is the first and key step of exerting pathogenicity for a bacterium [38], we show that the colonization of the knockout strains of $g \operatorname{tr} A$, gtrB and gtrII in the gut of $C$. elegans was significantly reduced compared with that of the wild type. The $g \operatorname{tr} A$ /B/II gene cluster optimizes the virulence of P. donghuensis HYS toward C. elegans through colonization increased in the gut of $C$. elegans. At present, there are no reports on the effect of GtrA, GtrB and GtrII on bacterial virulence resulting from changes in bacterial colonization in the host intestine.

Unlike higher organisms, C. elegans only has innate immune pathways, which play an important role in resisting pathogens and oxidative stress [39-41]. The MAPK, TGF- $\beta$ and DAF-2/DAF-16 signaling pathways are conserved innate immune signaling pathways 
necessary for $C$. elegans to resist pathogens. The p38 MAPK pathway belongs to the MAPK pathway, which is the most crucial pathway in intestinal innate immunity, making these pathways suitable for exploring the correlation between pathogenicity and host response [37]. TIR-1 (MAPKKK), SEK-1 (MAPKK) and PMK-1 (MAPK) pathways of the p38 MAPK pathway are critical in responding to infection [42]. In this study, we explored the defense response of $C$. elegans to a virulence infection with $P$. donghuensis HYS. We utilized the relevant mutant worms to conduct a slow-killing assay. Mutant ZD101 is deficient in the Toll/I-1 receptor (TIR-1) pathway, mutant KU4 is deficient in the SEK-1 pathway and mutant KU25 is deficient in the PMK-1 pathway. Mutant C. elegans died shortly after feeding on $P$. donghuensis HYS, but their viability was significantly improved when we used the $g \operatorname{tr} A B$ deletion mutant as an alternative food source. The results suggest a response to the $g \operatorname{tr} A / B / I I$-optimized virulence of $P$. donghuensis HYS that is mediated by the TIR-1, SEK-1 and PMK-1 components of the innate immune p38 MAPK pathway, which has not been previously reported. These pathways may supplement the virulence mechanism based on the $g \operatorname{tr} A / B / I I$ gene cluster.

In summary, we found that the specific virulence gene cluster $g \operatorname{tr} A / B / I I$ optimizes the virulence of $P$. donghuensis HYS toward $C$. elegans through colonization increased in the gut of C. elegans, and this process also involves C. elegans innate immunity. At present, there are no reports on the effect of GtrA, GtrB and GtrII on bacterial virulence resulting from changes in bacterial colonization in the host intestine, which suggests a novel virulence mechanism that differs from those used by other pathogenic Pseudomonas. Therefore, the $P$. donghuensis virulence phenotype differs from that of $P$. aeruginosa. These findings significantly expand upon the available information regarding $g \operatorname{tr} A / B / I I$-based virulence mechanisms in the genus Pseudomonas.

\section{Materials and Methods}

\subsection{Bacteria, Nematodes and Cultivation Conditions}

Tables S2 and S3 list the bacterial strains and plasmids used in this work, and Table S4 lists the primers. Escherichia coli strains and P. donghuensis strains were grown in LuriaBertani (LB) medium at $37^{\circ} \mathrm{C}$ and $30^{\circ} \mathrm{C}$, respectively. When necessary, antibiotics were added at the following final concentrations: for E. coli strains, $50 \mu \mathrm{g} / \mathrm{mL}$ kanamycin and $10 \mu \mathrm{g} / \mathrm{mL}$ gentamicin; for $P$. donghuensis strains, $25 \mu \mathrm{g} / \mathrm{mL}$ chloramphenicol, $50 \mu \mathrm{g} / \mathrm{mL}$ gentamicin and $50 \mu \mathrm{g} / \mathrm{mL}$ kanamycin.

All C. elegans strains were purchased from the Caenorhabditis Genetics Center (CGC, University of Minnesota, Twin Cities, USA) and maintained in standard conditions at 20 or $25^{\circ} \mathrm{C}$. The wild-type Bristol N2, ZD101 tir-1(qd4) III, KU4 sek-1(km4) X and KU25 pmk-1(km25) IV worms were obtained from the Caenorhabditis Genetic Center. C. elegans was sustained on nematode growth medium (NGM) agar plates with overnight cultures of E. coli OP50 [41] as the food source and then incubated for $8 \mathrm{~h}$ at $37^{\circ} \mathrm{C}$. To obtain synchronous day-1 adult worms, the eggs laid over half an hour were collected and grown at $22{ }^{\circ} \mathrm{C}$. Worm stocks were subjected to bleach treatment to remove contaminants [43], and worms from the generation after bleaching were used for experiments.

\subsection{Comparative Genomics Analysis}

The whole genome sequences of seven strains were processed and analysed by BGI (Shenzhen, China), and CD-HIT software was used for clustered, which enables the rapid clustering of similar proteins, with a threshold of $50 \%$ pairwise identity and a length difference cutoff of less than $30 \%$ amino acids. CD-HIT stands for Cluster Database at High Identity with Tolerance, the program takes a fasta format sequence database as input and produces a set of non-redundant (nr) representative sequences as output. In addition, CD-HIT outputs a cluster file, documenting the sequence groupies for each $\mathrm{nr}$ sequence representative [44,45]. These seven strains were P. donghuensis HYS (GenBank: AJJP01000001.1), P. aeruginosa PA14 (GenBank: CP000438.1), P. aeruginosa PAO1 (GenBank: AE004091.2), P. putida KT2440 (GenBank: AE015451.2), P. syringae B728a (GenBank: CP000075.1), P. syringae pv. tomato DC3000 (GenBank: 
AE016853.1) and P. fluorescens Pfo-1 (GenBank: CP000094.2). Gene families were constructed by integrating multiple software: the protein sequences were aligned in BLAST, the redundancy was eliminated by Solar, and gene family clustering treatment for the alignment results was carried out with Hcluster sg software. The sequences were processed and analyzed by BGI (Shenzhen, China). In addition, genes were aligned against several databases, including the NCBI nonredundant protein database (http://www.ncbi.nlm.nih.gov (accessed on $16 \mathrm{Au}-$ gust 2021)) and the KEGG pathway database (http://www.genome.jp/kegg (accessed on 16 August 2021)) by BLASTP.

\subsection{Screening of Virulence Factors}

The virulence factor database (VFDB, http://www.mgc.ac.cn/VFs/ (accessed on 16 August 2021)) is devoted to providing the scientific community with a comprehensive repository and online platform for deciphering bacterial pathogenesis. The virulence factor database (VFDB) is a comprehensive database integrating virulence factors of pathogenic bacteria. At present, it has the virulence gene sequence information of 30 genera (more than 100 kinds of medical pathogens). It involves 24 common pathogenic bacteria that are more important in medicine, including Pseudomonas, Shigella, Escherichia, Salmonella, and Bacillus. The prediction of virulence factors mainly depends on the specific software of the comparison algorithm; by using BLASTP against the VFDB, the comparison results can show the corresponding location of each gene annotated in the VFDB database and the description of virulence-related functions.

\subsection{Construction of Mutant and Complement P. donghuensis HYS}

Primers with restriction enzyme sites were designed to amplify fragments located upstream and downstream of each target gene (Table S4). A bacterial genome DNA purification kit (Promega, Madison, WI, USA), gel extraction DNA purification kit (Omega Bio-Tek, Norcross, GA, USA), plasmid DNA extraction kit (Omega Bio-Tek, Norcross, GA, USA) After two amplified fragments were digested with primer-specific restriction enzymes (quick restriction digestion enzymes purchased from Thermo Fisher Scientific) (Waltham, MA, USA) and ligated into the suicide vector $\mathrm{pEX} 18 \mathrm{Gm}$, the correct recombinant plasmid was transformed from E. coli S17-1 ( $\lambda$ pir) into P. donghuensis HYS via conjugation [46]. The target gene was knocked out, and selection for double recombinants was performed on LB agar plates containing 10\% $(\mathrm{wt} / \mathrm{vol})$ sucrose. The correct gene deletion mutants were further confirmed by PCR and sequencing. Related complementation mutants were constructed by ligating the Shine-Dalgarno sequences and open reading frames (ORFs) of the target genes into pBBR1MCS-2 [47]. After conjugation with the corresponding mutants, correct monoclonal targets were selected with double-antibiotic treatments and stored at $-80^{\circ} \mathrm{C}$.

\subsection{Construction of Point Mutations in P. donghuensis HYS}

Point mutations were introduced via homologous recombination using the suicide plasmid pEX18Gm. The amplified upstream and downstream fragments contained a homologous sequence of at least $20 \mathrm{bp}$, and the mutation site was in the homologous sequence (Table S4). The flanking segments were then in-frame ligated by fusion PCR and inserted into the suicide plasmid pEX18Gm with the restriction enzyme. Then, the correct recombinant plasmid was transformed from E. coli S17-1 ( $\lambda$ pir) into P. donghuensis HYS via conjugation [15], and the mutation site in the plasmid was inserted into the genome of P. donghuensis HYS. Finally, selection for double recombinants was performed on LB agar plates containing $10 \%(w / v)$ sucrose. The point mutants were further subject to PCR and sequencing to confirm they had the correct identity.

\subsection{Slow-Killing Assays of C. elegans}

In vivo infection assays and slow-killing assays of $C$. elegans were performed as previously described [15,48]. Approximately 100 synchronized adult worms were added to five plates and incubated at $22{ }^{\circ} \mathrm{C}$. C. elegans worms were considered dead or alive based on 
their response to being touched with a platinum wire. Abnormal deaths (crawling up the sides of the plates and drying out) were noted, and living C. elegans were transferred to new NGM plates every day. Worms were scored daily under an SZM-45B1 stereomicroscope (Sunny Optical, Yuyao, China) [49]. Kaplan-Meier survival curves were generated using the software IBM SPSS version 18.0 (SPSS Inc., Chicago, USA). Survival rates were compared and represented as the $\mathrm{LT}_{50}$ value. HYS or mutants were cultured in $\mathrm{LB}$ at $30{ }^{\circ} \mathrm{C}$ for $12 \mathrm{~h}$, and then transferred $200 \mu \mathrm{L}$ bacterial culture $\left(3 \times 10^{9} \mathrm{CFU} / \mathrm{mL}\right)$ to per NGM plate as food for C. elegans. The plates were dried before use. HYS was used as a positive control, and E. coli OP50, a traditional food source for C. elegans, was used as a negative control. Each experiment was replicated three to six times.

\subsection{Growth Curve Assay}

In order to eliminate the possible effect of strain growth on virulence, the growth curves of the strains on the NGM plate were determined [50,51]. Freshly isolated strains were inoculated in nutrient-rich LB liquid medium at $30^{\circ} \mathrm{C}$ and shaken and cultured for $12 \mathrm{~h}$. The $O D_{600}$ of the bacterial solution was determined and adjusted to be consistent, and $200 \mu \mathrm{L}$ of the bacterial solution was added to the plate. Three replicates were set for each strain. The strains on the NGM plate were grown at $22{ }^{\circ} \mathrm{C}$ for $12 \mathrm{~h}$, the concentration was appropriately diluted, and the growth curve was drawn.

\subsection{Transmission Electron Microscopy}

In order to reveal bacterial surface sugars, transmission electron microscopy was used at the highest possible resolution. Bacteria were washed three times and resuspended to an $O D_{600}$ of 0.2 . Bacteria were harvested and fixed in $3 \%(w / v)$ glutaraldehyde and $0.075 \%$ $(w / v)$ ruthenium red in $0.1 \mathrm{M}$ PBS for one hour in the dark, and they were post-fixed for two hours in the dark in $0.075 \%(w / v)$ ruthenium red and 1\% $(w / v)$ osmium tetroxide. Samples were dehydrated through graded ethanol, transferred to pure Spurr resin and blocked. Specimens were observed under transmission electron microscope (JEM-1400 plus, Japan Electronics Co. LTD, Shojima, Tokyo, Japan).

\subsection{C. elegans Bacterial CFU Analysis}

C. elegans were exposed to $P$. donghuensis HYS under the same conditions as used in the survival assay. Bacterial CFUs within the nematode gut were counted according to a method described in the literature [52]. After a defined period, 6 replicates of 10 worms each were transferred to M9 solution containing $25 \mathrm{mM}$ levamisole to paralyze the worm and stop pharyngeal pumping. Worms were washed twice with an antibiotic solution containing chloramphenicol $(25 \mu \mathrm{g} / \mathrm{mL})$ in levamisole $(25 \mathrm{mM})$, followed by $1 \mathrm{~h}$ of incubation in the antibiotic solution to kill bacteria present on the exterior of the worm. After three washes with a solution of $25 \mathrm{mM}$ levamisole to remove the antibiotics, worms were lysed with a motorized pestle. Lysates were serially diluted in M9 solution and plated on Luria-Bertani plates containing chloramphenicol $(25 \mu \mathrm{g} / \mathrm{mL})$ to select for P. donghuensis HYS and select against OP50. After overnight incubation at $30^{\circ} \mathrm{C}$, colonies of P. donghuensis HYS were counted to determine CFU per worm.

\subsection{Microscopy}

Experiments involving worms were performed by utilizing the SZM-45B1 stereomicroscope (Sunny Optical, Yuyao, China).

\subsection{Statistical Analysis}

All data were presented as the means \pm standard deviation (SD), and each experiment was performed at least three times independently. Survival curves were plotted in IBM SPSS version 18.0 (SPSS Inc., Chicago, USA) using the Kaplan-Meier method. Statistical analyses were performed using OriginPro 9.0 (OriginLab, USA). Significant differences were evaluated using the Student's $t$-test, $p<0.05$ was considered statistically significant. 


\subsection{Accession Numbers}

The GenBank accession numbers for the genes $g \operatorname{tr} A$, gtrB, and gtrII from $P$. donghuensis HYS are UW3_RS0104075, UW3_RS0104080, UW3_RS26470, respectively.

Supplementary Materials: The following are available online at https://www.mdpi.com/article/10 $.3390 /$ ijms221910741/s1.

Author Contributions: Y.X. designed and performed the experiments, performed the statistical analysis and made most of the figures and tables, and wrote the manuscript. P.W. and X.Z. performed some of the experiments and made the corresponding figures and tables. Z.X. contributed to conceptualization and funding acquisition. All authors have read and agreed to the published version of the manuscript.

Funding: Please add: This research was funded by the National Natural Science Foundation of China (No. 31570090, No. 31800028). This project was partially supported by National Infrastructure of Natural Resources for Science and Technology Program of China (number NIMR-2021-12).

Institutional Review Board Statement: Not applicable.

Informed Consent Statement: Not applicable.

Data Availability Statement: All data generated or analysed during this study are included in this published article (and its Supplementary Information files).

Acknowledgments: The worm strains used in this study were provided by the CGC.

Conflicts of Interest: The authors declare no conflict of interest.

\section{References}

1. Hornef, M.W.; Wick, M.J.; Rhen, M.; Normark, S. Bacterial strategies for overcoming host innate and adaptive immune responses. Nat. Immunol. 2002, 3, 1033-1040. [CrossRef]

2. Rohmer, L.; Hocquet, D.; Miller, S.I. Are pathogenic bacteria just looking for food? Metabolism and microbial pathogenesis. Trends Microbiol. 2011, 19, 341-348. [CrossRef] [PubMed]

3. Wang, H.R.; Hu, Y.H.; Zhang, W.W.; Sun, L. Construction of an attenuated Pseudomonas fluorescens strain and evaluation of its potential as a cross-protective vaccine. Vaccine 2009, 27, 4047-4055. [CrossRef] [PubMed]

4. Fernandez, M.; Porcel, M.; de la Torre, J.; Molina-Henares, M.A.; Daddaoua, A.; Llamas, M.A.; Roca, A.; Carriel, V.; Garzon, I.; Ramos, J.L.; et al. Analysis of the pathogenic potential of nosocomial Pseudomonas putida strains. Front. Microbiol. 2015, 6, 871-881. [CrossRef] [PubMed]

5. Al-Wrafy, F.; Brzozowska, E.; Gorska, S.; Gamian, A. Pathogenic factors of Pseudomonas aeruginosa-The role of biofilm in pathogenicity and as a target for phage therapy. Postep. Hig. Med. Dosw. 2017, 71, 78-91. [CrossRef] [PubMed]

6. Xin, X.F.; He, S.Y. Pseudomonas syringae pv. Tomato DC3000: A model pathogen for probing disease susceptibility and hormone signaling in plants. Annu. Rev. Phytopathol. 2013, 51, 473-498. [CrossRef]

7. Hoiby, N. Pseudomonas aeruginosa infection in cystic fibrosis. Relationship between mucoid strains of Pseudomonas aeruginosa and the humoral immune response. Acta Pathol. Microbiol. Scand. 1974, 82, 551-558. [CrossRef]

8. Reynolds, H.Y.; Levine, A.S.; Wood, R.E.; Zierdt, C.H.; Dale, D.C.; Pennington, J.E.; Maryland, B. Pseudomonas aeruginosa infections: Persisting problems and current research to find new therapies. Ann. Intern. Med. 1975, 82, 819-831. [CrossRef] [PubMed]

9. Dzvova, N.; Colmer-Hamood, J.A.; Griswold, J.A.; Hamood, A.N. Isolation and characterization of HepP: A virulence-related Pseudomonas aeruginosa heparinase. BMC Microbiol. 2017, 17, 233-248. [CrossRef]

10. Ossowicki, A.; Jafra, S.; Garbeva, P. The antimicrobial volatile power of the rhizospheric isolate Pseudomonas donghuensis P482. PLoS ONE 2017, 12, e0174362. [CrossRef]

11. Agaras, B.C.; Iriarte, A.; Valverde, C.F. Genomic insights into the broad antifungal activity, plant-probiotic properties, and their regulation, in Pseudomonas donghuensis strain SVBP6. PLoS ONE 2018, 13, e0194088. [CrossRef]

12. Gao, J.; Yu, X.; Xie, Z. Draft genome sequence of high-siderophore-yielding Pseudomonas sp. strain HYS. J. Bacteriol. 2012, 194, 4121. [CrossRef] [PubMed]

13. Gao, J.; Xie, G.; Peng, F.; Xie, Z. Pseudomonas donghuensis sp. nov., exhibiting high-yields of siderophore. Antonie Van Leeuwenhoek 2015, 107, 83-94. [CrossRef] [PubMed]

14. Yu, X.Y.; Chen, M.; Jiang, Z.; Hu, Y.; Xie, Z.X. The two-component regulators GacS and GacA positively regulate a nonfluorescent siderophore through the Gac/Rsm signaling cascade in high-siderophore-yielding Pseudomonas sp. Strain HYS. J. Bacteriol. 2014, 196, 3259-3270. [CrossRef] [PubMed]

15. Xie, G.F.; Zeng, M.; You, J.; Xie, Z.X. Pseudomonas donghuensis HYS virulence towards Caenorhabditis elegans is regulated by the Cbr/Crc system. Sci. Rep. 2019, 9, 8772. [CrossRef] [PubMed] 
16. Gui, Z.; You, J.; Xie, G.; Qin, Y.; Wu, T.; Xie, Z. Pseudomonas donghuensis HYS 7-hydroxytropolone contributes to pathogenicity toward Caenorhabditis elegans and is influenced by pantothenic acid. Biochem. Biophys. Res. Commun. 2020, 533, 50-56. [CrossRef] [PubMed]

17. Shen, P.; Yue, Y.; Park, Y. A living model for obesity and aging research: Caenorhabditis elegans. Crit. Rev. Food Sci. Nutr. 2018, 58, 741-754. [CrossRef] [PubMed]

18. Corsi, A.K.; Wightman, B.; Chalfie, M. A Transparent window into biology: A primer on Caenorhabditis elegans. Genetics 2015, 200, 387-407. [CrossRef]

19. O'Reilly, L.P.; Luke, C.J.; Perlmutter, D.H.; Silverman, G.A.; Pak, S.C. C. elegans in high-throughput drug discovery. Adv. Drug Deliv. Rev. 2014, 69, 247-253. [CrossRef]

20. Markaki, M.; Tavernarakis, N. Modeling human disease in Caenorhabditis elegans. Biotechnol. J. 2010, 5, 1261-1276. [CrossRef]

21. Sorathia, N.; Rajadhyaksha, M.S. Caenorhabditis elegans: A Model for Studying Human Pathogen Biology. Recent Pat. Biotechnol. 2016, 10, 217-225. [CrossRef] [PubMed]

22. Brenner, S. The genetics of Caenorhabditis elegans. Genetics 1974, 77, 71-94. [CrossRef] [PubMed]

23. Garbeva, P.; Veen, J.A.; Elsas, J.D. Assessment of the diversity, and antagonism towards Rhizoctonia solani AG3, of Pseudomonas species in soil from different agricultural regimes. FEMS Microbiol. Ecol. 2004, 47, 51-64. [CrossRef]

24. Knirel, Y.A.; Sun, Q.; Senchenkova, S.N.; Perepolov, A.V.; Shashkov, A.S.; Xu, J. O-antigen modifications providing antigenic diversity of Shigella flexneri and underlying genetic mechanisms. Biochemistry 2015, 80, 901-914. [CrossRef]

25. Kolly, G.S.; Mukherjee, R.; Kilacskova, E.; Abriata, L.A.; Raccaud, M.; Blasko, J.; Sala, C.; Dal, P.M.; Mikusova, K.; Cole, S.T. GtrA protein RV3789 is required for arabinosylation of arabinogalactan in Mycobacterium tuberculosis. J. Bacteriol. 2015, 197, 3686-3697. [CrossRef]

26. Ardiccioni, C.; Clarke, O.B.; Tomasek, D.; Issa, H.A.; von Alpen, D.C.; Pond, H.L.; Banerjee, S.; Rajashankar, K.R.; Liu, Q.; Guan, Z.; et al. Structure of the polyisoprenyl-phosphate glycosyltransferase GtrB and insights into the mechanism of catalysis. Nat. Commun. 2016, 7, 10175-10183. [CrossRef]

27. Okada, N.; Sasakawa, C.; Tobe, T.; Yamada, M.; Nagai, S.; Talukder, K.A.; Komatsu, K.; Kanegasaki, S.; Yoshikawa, M. Virulenceassociated chromosomal loci of Shigella flexneri identified by random Tn5 insertion mutagenesis. Mol. Microbiol. 1991, 5, 187-195. [CrossRef]

28. Guan, S.; Bastin, D.A.; Verma, N.K. Functional analysis of the O antigen glucosylation gene cluster of Shigella flexneri bacteriophage SfX. Microbiology 1999, 145, 1263-1273. [CrossRef] [PubMed]

29. Mavris, M.; Manning, P.A.; Morona, R. Mechanism of bacteriophage SfII-mediated serotype conversion in Shigella flexneri. Mol. Microbiol. 1997, 26, 939-950. [CrossRef] [PubMed]

30. Burton, N.O.; Riccio, C.; Dallaire, A.; Price, J.; Jenkins, B.; Koulman, A.; Miska, E.A. Cysteine synthases CYSL-1 and CYSL-2 mediate C. elegans heritable adaptation to P. vranovensis infection. Nat. Commun. 2020, 11, 1741-1754. [CrossRef] [PubMed]

31. Maier, C.; Huptas, C.; von Neubeck, M.; Scherer, S.; Wenning, M.; Lücking, G. Genetic Organization of the aprX-lipA2 Operon Affects the Proteolytic Potential of Pseudomonas Species in Milk. Front. Microbiol. 2020, 11, 1190-1203. [CrossRef]

32. Khan, A.H.; Anees, M.; Arshad, M.; Muhammad, Y.S.; Iqbal, M.; Yousaf, S. Effects of illuminance and nutrients on bacterial photo-physiology of hydrocarbon degradation. Sci. Total Environ. 2016, 557, 705-711. [CrossRef]

33. Tribelli, P.M.; Raiger Iustman, L.J.; Catone, M.V.; Di Martino, C.; Revale, S.; Méndez, B.S.; López, N.I. Genome sequence of the polyhydroxybutyrate producer Pseudomonas extremaustralis, a highly stress-resistant Antarctic bacterium. J. Bacteriol. 2012, 194, 2381-2382. [CrossRef] [PubMed]

34. Lehane, A.M.; Korres, H.; Verma, N.K. Bacteriophage-encoded glucosyltransferase GtrII of Shigella flexneri: Membrane topology and identification of critical residues. Biochem. J. 2005, 389, 137-1433. [CrossRef] [PubMed]

35. West, N.P.; Sansonetti, P.; Mounier, J.; Exley, R.M.; Parsot, C.; Guadagnini, S.; Prevost, M.C.; Prochnicka-Chalufour, A.; Delepierre, M.; Tanguy, M.; et al. Optimization of virulence functions through glucosylation of Shigella LPS. Science 2005, 307, $1313-1317$. [CrossRef] [PubMed]

36. Clement, M.J.; Imberty, A.; Phalipon, A.; Perez, S.; Simenel, C.; Mulard, L.A.; Delepierre, M. Conformational studies of the O-specific polysaccharide of Shigella flexneri 5 a and of four related synthetic pentasaccharide fragments using NMR and molecular modeling. J. Biol. Chem. 2003, 278, 47928-47936. [CrossRef] [PubMed]

37. Blocker, A.; Gounon, P.; Larquet, E.; Niebuhr, K.; Cabiaux, V.; Parsot, C.; Sansonetti, P. The tripartite type III secreton of Shigella flexneri inserts IpaB and IpaC into host membranes. J. Cell. Biol. 1999, 147, 683-693. [CrossRef]

38. Kusmierek, M.; Dersch, P. Regulation of host-pathogen interactions via the post-transcriptional Csr/Rsm system. Curr. Opin. Microbiol. 2018, 41, 58-67. [CrossRef] [PubMed]

39. Pukkila-Worley, R.; Ausubel, F.M. Immune defense mechanisms in the Caenorhabditis elegans intestinal epithelium. Curr. Opin. Immunol. 2012, 24, 3-9. [CrossRef] [PubMed]

40. Sansonetti, P.J. Pathogenesis of shigellosis: From molecular and cellular biology of epithelial cell invasion to tissue inflammation and vaccine development. Jpn. J. Med. Sci. Biol. 1998, 51, S69-S80. [CrossRef]

41. Bernardes, E.V.T.; Charron-Mazenod, L.; Reading, D.J.; Reckseidler-Zenteno, S.L.; Lewenza, S. Exopolysaccharide-Repressing Small Molecules with Antibiofilm and Antivirulence Activity against Pseudomonas aeruginosa. Antimicrob. Agents Chemother. 2017, 61, e01997-16. [CrossRef] 
42. Huang, X.; Pan, W.; Kim, W.; White, A.; Li, S.; Li, H.; Lee, K.; Fuchs, B.B.; Zeng, K.; Mylonakis, E. Caenorhabditis elegans mounts a p38 MAPK pathway-mediated defence to Cutibacterium acnes infection. Cell. Microbiol. 2020, 22, e13234. [CrossRef]

43. Nystul, T.G.; Roth, M.B. Carbon monoxide-induced suspended animation protects against hypoxic damage in Caenorhabditis elegans. Proc. Natl. Acad. Sci. USA 2004, 101, 9133-9136. [CrossRef] [PubMed]

44. Hoang, T.T.; Karkho-Schweizer, R.R.; Kutchma, A.J.; Schweizer, H.P. A broad-host-range Flp-FRT recombination system for site-specific excision of chromosomally-located DNA sequences: Application for isolation of unmarked Pseudomonas aeruginosa mutants. Gene 1998, 212, 77-86. [CrossRef]

45. Meisel, J.D.; Panda, O.; Mahanti, P.; Schroeder, F.C.; Kim, D.H. Chemosensation of bacterial secondary metabolites modulates neuroendocrine signaling and behavior of C. elegans. Cell 2014, 159, 267-280. [CrossRef]

46. Fu, L.; Niu, B.; Zhu, Z.; Wu, S.; Li, W. CD-HIT: Accelerated for clustering the next-generation sequencing data. Bioinformatics 2012, 28, 3150-3152. [CrossRef]

47. Li, W.; Godzik, A. Cd-hit: A fast program for clustering and comparing large sets of protein or nucleotide sequences. Bioinformatics 2006, 22, 1658-1659. [CrossRef] [PubMed]

48. Simon, R.; Priefer, U.; Puhler, A. A broad host range mobilization system for in vivo genetic engineering transposon mutagenesis in gram negative bacteria. Bio/Technology 1983, 1, 784-791. [CrossRef]

49. Kovach, M.E.; Elzer, P.H.; Hill, D.S.; Robertson, G.T.; Farris, M.A.; Roop, R.M.; Peterson, K.M. Four new derivatives of the broad-host-range cloning vector pBBR1MCS, carrying different antibiotic-resistance cassettes. Gene 1995, 166, 175-176. [CrossRef]

50. Tan, M.W.; Mahajan-Miklos, S.; Ausubel, F.W. Killing of Caenorhabditis elegans by Pseudomonas aeruginosa used to medel mammalian bacterial pathogenesis. Proc. Natl. Acad. Sci. USA 1999, 96, 715-720. [CrossRef]

51. Hansen, M.; Taubert, S.; Crawford, D.; Libina, N.; Lee, S.-J.; Kenyon, C. Lifespan extension by conditions that inhibit translation in Caenorhabditis elegans. Aging Cell 2007, 6, 95-110. [CrossRef] [PubMed]

52. Rezzoagli, C.; Granato, E.T.; Kummerli, R. In-vivo microscopy reveals the impact of Pseudomonas aeruginosa social interactions on host colonization. ISME J. 2019, 13, 2403-2414. [CrossRef] [PubMed] 